\title{
The assessment and management of gamma hydroxybutyrate use in general practice
}

Vicky Phan, Shalini Arunogiri, Dan I Lubman

\section{Background \\ Gamma hydroxybutyrate (GHB) is an illicit drug commonly used in music festival, party and 'chemsex' settings. Most people who use GHB do so occasionally, without dependent use or withdrawal symptoms. However, a minority of users experience harms including unconsciousness and respiratory collapse in overdose. Adverse interactions can also occur when GHB is used with other drugs (eg methamphetamine), necessitating assessment, management or onward referral by general practitioners.}

\section{Objective}

This article describes the use of $\mathrm{GHB}$, with a contemporary update on principles of assessment and management in general practice, brief intervention and harm-minimisation strategies, and indicators for referral to a specialist in dependent use.

\section{Discussion}

The assessment and management of individuals with GHB-related harms in general practice is supported by an awareness of the context of use, familiarity with targeted harmminimisation advice and cognisance of markers of risk indicating onward referral to specialist addiction services when appropriate.
GAMMA HYDROXYBUTYRATE (GHB) is an illicit drug that has a depressant effect on the central nervous system (CNS). ${ }^{1}$ $\mathrm{GHB}$ is associated with a disproportionate burden of harm despite low overall population prevalence of use, with GHB-related harms frequently requiring acute health intervention or alcohol and other drug (AOD) treatment..$^{2,3}$ Individuals may therefore present to their general practitioners (GPs) for discharge follow-up and onward care planning. This article presents a concise summary of literature supporting best-practice clinical assessment and management of GHB tailored to a general practice setting, drawn from recently updated evidencebased guidelines. ${ }^{1}$

\section{What is gamma hydroxybutyrate?} GHB use was initially reported in Europe, the USA and Australia during the 1990s, and it continues to be consumed in recreational nightlife settings for its alcohol-like effects. ${ }^{4}$ Approximately $1 \%$ of Australians have ever used GHB, and $0.1 \%$ report having used it in the past year. ${ }^{5}$ The health costs associated with GHB consumption are relatively high when compared with other drugs, despite this low prevalence of use. This is due to rising rates of presentations to AOD treatment, detoxification services, and ambulance and emergency department settings for GHB intoxication or associated injuries (eg falls and assaults). ${ }^{2,3}$
In Australia, GHB is commonly used within the dance and party scene, and by men who have sex with men (MSM). ${ }^{6,7}$ GHB may be used before or during sex to increase sexual pleasure ('chemsex') because of its relaxing, sexuallystimulating and euphoric effects at low doses. ${ }^{2,8}$ Street names include 'G', 'liquid ecstasy', 'liquid E', 'grievous bodily harm' and 'scoop'. ${ }^{1}$ GHB is usually available as a bitter or salty clear, odourless liquid, distributed in small bottles or vials. GHB can also be presented as a bright blue liquid known as 'blue nitro', and less frequently is available as a powder. ${ }^{4}$ The purity and concentration of liquid containing GHB can also vary, making the monitoring of dosing difficult. GHB is typically swallowed but it can also be injected, insufflated or inserted rectally. ${ }^{1,6}$ There are also increasing concerns about the recreational use of two industry chemicals, gamma-butyrolactone (GBL) and 1,4-butanediol (1,4-BD), which quickly convert to GHB when ingested., ${ }^{4,9}$ These are consumed in liquid form and have a more bitter and unpleasant taste than GHB.

GHB acts primarily on gammaaminobutyric acid receptors but also exerts effects on dopamine, serotonin and cholinergic neurotransmission..$^{1,4}$ It is primarily a CNS depressant but at low doses can produce euphoric effects and effects similar to those of stimulants. ${ }^{6}$ The effects of GHB start approximately 15-20 minutes after it is taken and can 
last up to four hours. ${ }^{10,11}$ Individuals typically take small doses of GHB multiple times in a session, as it has a half-life of only 20-30 minutes. People who are dependent on GHB are likely to use GHB more regularly (eg every four hours) and over prolonged periods. ${ }^{12}$ Individuals who use GHB also tend to concurrently use other drugs, including alcohol, amphetamine-type stimulants (eg methamphetamine), ketamine and cannabis. ${ }^{4}$ This is significant because GHB's main risk is its potential for overdose, particularly when taken with other CNS depressant drugs. ${ }^{1,6,11}$ Use of GHB with other drugs also leads to an increased risk for withdrawal complications. ${ }^{1}$

\section{Intoxication, dependence and withdrawal}

Desired effects of GHB intoxication include euphoria, relaxation, increased libido, sociability and reduced inhibitions. ${ }^{4,10,13-15}$ Negative effects include amnesia, drowsiness, confusion, tremor, bradycardia, vomiting, diarrhoea, urinary incontinence, agitation, anxiety, paranoia and psychosis. ${ }^{1,2,6,8}$ When GHB is used in conjunction with a stimulant (eg methamphetamine), the risk of experiencing a seizure increases. ${ }^{1}$
Overdoses are common among people who use GHB, partly because of its steep dose-response curve, which means that there is a small difference between the amount of $\mathrm{GHB}$ required to become intoxicated and the amount of GHB that can result in overdose. ${ }^{6,16}$ In an Australian study of 76 people who used GHB, half had a history of overdose during which they lost consciousness. ${ }^{2}$ Overdoses typically occur as a consequence of using large concentrations of GHB over a short period, or when GHB is used in combination with other CNS depressants (eg alcohol or benzodiazepines)..$^{1,6,11}$ Symptoms of a GHB overdose include shallow or irregular breathing, confusion, hallucinations, agitation, seizures, blackouts, coma and death. ${ }^{1,6}$ The usual clinical course following a GHB overdose - if other sedative hypnotics were not concurrently used - is rapid, spontaneous awakening from GHB-induced loss of consciousness or coma. ${ }^{6} \mathrm{CNS}$ depression usually persists for 1-3 hours, with individuals typically making a full and uneventful recovery within 4-8 hours. ${ }^{6,17-20}$

The symptoms of GHB withdrawal (Table 1) are similar to those of alcohol withdrawal but with a more sudden onset within the first 24 hours of cessation. ${ }^{6,11}$ Withdrawal symptoms are typically most pronounced on the first day and

\section{Table 1. Symptoms of gamma hydroxybutyrate withdrawa ${ }^{1,6}$}

\begin{tabular}{ll}
\hline Withdrawal stage & Symptoms \\
\hline Less than 24 hours since last & Insomnia \\
gamma hydroxybutyrate use & Anxiety \\
& Restlessness \\
& Tremor \\
& Sweating \\
& Tachycardia \\
& Nausea \\
& Vomiting \\
\hline More than 24 hours since last & Transient tachycardia and hypertension \\
gamma hydroxybutyrate use & Agitation and combativeness \\
& Visual, auditory, olfactory and tactile hallucinations \\
& Autonomic instability \\
& Seizures \\
& Delusions \\
& Paranoia \\
& Delirium \\
\hline
\end{tabular}

can persist for up to two weeks, with symptoms waxing and waning during this time. ${ }^{6}$ While withdrawal symptoms can be self-limiting for some patients, a minority of individuals are at risk of a more severe GHB withdrawal that can progress to delirium. ${ }^{1}$ Delirium is more likely for individuals who use GHB more frequently in large amounts or concurrently with stimulants or other CNS depressants. ${ }^{1,6,21}$ GHB withdrawal can occur after as little as 2-3 months of use, or following even shorter time frames where there has been high-frequency use (eg every 30 minutes to every three hours). ${ }^{6,21}$ Doses as low as $18 \mathrm{~g}$ /day have been associated with the manifestation of withdrawal symptoms. ${ }^{21}$

GHB dependence is suggested by daily use multiple times throughout the day, waking overnight to use GHB, symptoms of withdrawal on days of abstinence and using other drugs to prevent GHB withdrawal symptoms. A tool to screen for dependence is provided in Table $2 .^{22}$

\section{Assessing gamma hydroxybutyrate use}

The majority of people who use GHB do so in an occasional, recreational pattern of use, and may not present with symptoms of dependence or withdrawal. Therefore, a thorough assessment (refer to Table 2) is necessary to determine how to appropriately offer tailored advice, ranging from education, harm reduction and brief intervention for most individuals, to a management plan involving referral options for those with symptoms of dependence. ${ }^{22}$ It is recommended to specifically ask about other drug use, particularly concurrent CNS depressant use, which can complicate management. Individuals benefiting from referral to specialist AOD services include those with risk factors for complex withdrawal, such as previous complicated withdrawal, and frequent and high dose GHB use. ${ }^{1,6}$

\section{Managing gamma hydroxybutyrate use}

\section{Brief intervention}

All individuals presenting with GHB use may benefit from a brief intervention 
using the feedback, responsibility, advice, menu of options, empathy and self-efficacy (FRAMES) approach in Table $3 .^{23}$

\section{Harm minimisation}

Individuals who plan to continue using GHB should be advised to adopt harm-minimisation strategies. This may include taking a smaller test dose first, setting limits on the quantity used in a session, only using GHB in safe places, telling someone what they have taken, avoiding using AOD before or at the same time and reducing the quantity of GHB taken when combining drugs. ${ }^{24}$ Individuals who use GHB should also be reminded of safe sexual practices and may be provided with the 'S-T-A-Y-I-N-G S-A-F-E' proforma in Table $4 .^{6}$

\section{Gamma hydroxybutyrate withdrawal management}

AOD specialist input is advised when planning support for GHB dependence (refer to Resources), as withdrawal can be associated with medical and psychiatric complications. A reducing regimen of benzodiazepines (eg diazepam) is the standard pharmacotherapy approach used for the management of GHB withdrawal. For mild withdrawal this is often adequate, in the context of a low-stimulus environment, adequate supportive care, nutrition and hydration. ${ }^{1,25}$

Some individuals may require referral to AOD specialist services for withdrawal support in either home or residential

\section{Table 2. Assessment of gamma hydroxybutyrate use}

Screening for GHB dependence (the presence of $\geq 2$ of these features within a 12-month period suggests dependence $)^{22}$
Assessment of the individual's past experience and management of GHB withdrawal and use

\begin{tabular}{llc} 
& Yes & No \\
\hline Strong urges or desires to use & $\square$ & $\square$ \\
\hline Difficulties controlling use & $\square$ & $\square$ \\
\hline Use of excessive amounts & $\square$ & $\square$ \\
\hline Diminishing effect obtained from usual dose & $\square$ & $\square$ \\
\hline Use over a longer period of time than intended & $\square$ & $\square$ \\
\hline Use to relieve or avoid withdrawal symptoms & $\square$ & $\square$ \\
\hline Use despite related physical or psychological complications & $\square$ & $\square$ \\
\hline Diminished ability to function in activities due to use & $\square$ & $\square$ \\
\hline Failure to fulfil necessary obligations at work, school or home & $\square$ & $\square$ \\
\hline Social problems or relationship difficulties caused by use & $\square$ & $\square$ \\
\hline Use results in exposure to situations that are hazardous & $\square$ & $\square$ \\
\hline $\begin{array}{l}\text { Quantity used? } \\
\text { Frequency of use? }\end{array}$ & & \\
Route of administration? & & \\
Setting of use? & & \\
Reasons for use? & &
\end{tabular}

Any history of complicated withdrawal from GHB use (eg delirium, seizures)?

Past withdrawal symptoms experienced?

How has withdrawal previously been managed?

Any past interactions with emergency services resulting from GHB use?

Any past interactions with drug and alcohol services for GHB use?

What was the outcome of previous withdrawal and treatment?

Consider using a screening instrument such as the World Health Organization Alcohol, Smoking and Substance Involvement Screening Test.

or dependence

Does the individual have any comorbid physical issues?

Does the individual have any comorbid psychiatric issues?

Assessment of pregnancy status
Women using GHB should be screened for pregnancy, which would complicate management and warrant referral to specialist AOD services. 
settings. Individuals who have histories of complicated GHB withdrawal or high-frequency, high-dose use would be best advised to undertake withdrawal in a supervised setting. The prolonged and potentially severe symptoms that can be experienced by people who are dependent on GHB may lead to a poor outcome if conducted at home - either as a consequence of possible medical complications or overdose if individuals attempt to relieve their withdrawal symptoms too early with alcohol and/or benzodiazepines. ${ }^{1}$ Similarly, individuals

\section{Table 3. Feedback, responsibility, advice, menu of options, empathy and self-efficacy (FRAMES) approach ${ }^{23}$}

\begin{tabular}{ll}
\hline Feedback & $\begin{array}{l}\text { Provide feedback to the individual about the risks of their GHB use and } \\
\text { identify any risk factors for severe withdrawal }\end{array}$ \\
\hline Responsibility & $\begin{array}{l}\text { Reinforce that any decision to address the GHB use (or not) lies with } \\
\text { the user }\end{array}$ \\
\hline Advice & $\begin{array}{l}\text { Offer a simple and direct assessment of the impact GHB appears to be } \\
\text { having on the individual, advise on the risks of continued use and offer } \\
\text { your advice to the individual to address this }\end{array}$ \\
\hline Menu & $\begin{array}{l}\text { Provide a menu of options for the individual to manage their GHB use } \\
\text { Empathy }\end{array}$ \\
\hline Colf-efficacy & Encourage the individual to believe they can change \\
\hline GHB, gamma hydroxybutyrate
\end{tabular}

\section{Table 4. STAYING SAFE ${ }^{6}$}

S Seek medical attention immediately if you have taken too much GHB. Do not use other drugs in the hope of reversing the effects.

$\mathrm{T} \quad$ Two or more substances used at the same time increase the risk of overdose significantly (especially sedatives; eg alcohol, ketamine).

A Always measure GHB doses accurately (eg with a syringe or pipette). Wait until the effects are felt and do not re-dose for at least two hours.

Y You should always avoid using GHB on your own and always use in a safe place and with someone who has not taken it, as it is common to become unconscious.

I If you have used and are going to sleep, sleep on your side in case you are sick. Place sleeping or unconscious friends in the recovery position.

$\mathrm{N} \quad$ Never keep GHB in drink bottles, where it might be drunk by others not aware of the content. Add food colouring to avoid accidental drinking.

G GHB is addictive and dependence can happen quickly. Avoid frequent use, especially daily use.

S Severe and potentially serious GHB withdrawal symptoms occur if you are dependent and you miss a dose or reduce amounts taken abruptly.

A Acute withdrawal symptoms and have no GHB? Seek medical help immediately in an emergency department.

F $\quad$ Find medical support for planned GHB detoxification. Do not attempt to stop abruptly on your own. If you want to reduce your dose, do so in very small doses until you find medical support.

E Employ methods to stabilise your use; consumption diaries can be helpful.

who are dependent on GHB should be advised not to abruptly cease their use or attempt an unsupported detoxification. If an individual who uses GHB has any symptoms of complicated withdrawal or delirium, they should be immediately transferred to a tertiary hospital for inpatient management with AOD specialist support.

\section{Gamma hydroxybutyrate withdrawal management in individuals with co-occurring mental illness}

A mental illness is not a contraindication to GHB withdrawal, especially when an individual's use of GHB is putting their health at risk. ${ }^{1}$ There can, however, be a complex and bi-directional relationship between GHB use and withdrawal and mental health problems. GHB use and withdrawal may manifest as, or worsen, psychiatric symptoms. ${ }^{3}$ Anxiety and psychotic symptoms have been observed during the acute withdrawal period, and if these interfere with functioning or cause risk to the individual or others, referral to specialist mental health services may be required. Conversely, the presence of mental illness may increase the complexity of care required during withdrawal. ${ }^{1}$ Assessment by specialist AOD services with access to mental health input (eg within 'dual diagnosis' clinics) is therefore recommended for the coordination of care of these individuals. Furthermore, if active mental health symptoms are present, the advantages and disadvantages of commencing withdrawal should be considered with the individual and their treating mental health clinician or team.

\section{Post-withdrawal care planning}

It is vital to have a discussion about the increased risk of overdose resulting from reduced tolerance to GHB after any GHB withdrawal. ${ }^{1}$

Post-withdrawal care planning should commence prior to withdrawal and be led by the individual. This may include referral to AOD services for relapse prevention counselling, management of other additional substance use, and review and management of possible underlying mental health issues or psychosocial stressors. 
Post-withdrawal care aims to support the individual's goals relating to substance-use recovery. A holistic approach is recommended, with consideration of the individual's safety and protection, accommodation stability, financial stability, capacity to work, relationships and any concurrent legal or child-protection issues. Where possible, any significant others should be encouraged to support the individual and help implement the post-withdrawal plan.

\section{Families/significant others}

Consideration should be given to the needs of family and significant others when managing an individual who uses GHB. Where appropriate, information should be provided to the family and significant others regarding the GHB withdrawal process and support services available to them.

\section{Resources}

Patient factsheets about GHB use are available from the following organisations:

- Alcohol and Drug Foundation (https://adf.org.au/drug-facts/ghb/)

- TouchBase (https://touchbase.org.au/ alcohol-and-drugs/ghbgbl).

GPs wishing to speak with an AOD specialist for secondary advice may contact their local AOD service or their state or territory telephone AOD specialist consultancy service. These numbers are not for patients and are for use by health professionals only:

- New South Wales/Australian Capital Territory: Drug and Alcohol Specialist Advisory Service (DASAS) (02) 93618006

- Victoria: Drug and Alcohol Clinical Advisory Service (DACAS VIC) 1800812804

- Queensland: Alcohol and Drug Clinical Advisory Service (ADCAS) 1800290928

- South Australia: Drug and Alcohol Clinical Advisory Service (DACAS) (08) 70871742

- Tasmania: DACAS VIC - 1800630093

- Northern Territory: DACAS VIC 1800111092

- Western Australia: Clinical Advisory Service - (08) 94425042.
Wherever possible, individuals who identify as lesbian, gay, bisexual, trans and gender diverse, intersex and queer (LGBTIQ) should be referred to a health promotion organisation that reinforces a message of LGBTIQ inclusivity and acceptance. A referral database is available from Q Life, the national counselling and referral service for LGBTIQ people: Q Life - 1800184527.

\section{CASE}

James, aged 23 years, is a man who presents to you after a presentation to the emergency department (ED) on the weekend. He was transported to the ED by ambulance when his partner, Anne, became concerned by his uncharacteristic jealousy and erratic behaviour. When assessed in the ED, James admitted to having used 'ice' (methamphetamine) and GHB. He acknowledged a tendency to become transiently concerned about Anne's fidelity when smoking methamphetamine, but remained able to challenge this thought and denied any intention to harm Anne. James settled after being administered $10 \mathrm{mg}$ diazepam and observed for few hours in the ED. The discharge summary recommends follow-up and management planning for his methamphetamine and GHB use. James is also seeking to cease his substance use as he is concerned that his recent jealousy while intoxicated is straining his relationship with Anne.

You assess James using Table 2 and identify that James has been smoking $0.1 \mathrm{~g}$ methamphetamine and swallowing up to $10 \mathrm{~mL} \mathrm{GHB}$ when out clubbing on weekends. He first started using these drugs six months ago and does not meet the criteria for dependence. His use has been perpetuated by his enjoyment of the 'buzz' and euphoria he experiences when intoxicated on methamphetamine and GHB. He describes at least one unintentional GHB overdose in the past when his friends discovered him 'sleeping' in a corner at a party after using GHB. He does not use GHB during the week when he works as a labourer and does not have any craving. James has never previously sought addiction treatment, has no comorbid physical or mental health issues, and would consider drug and alcohol counselling.

Your assessment suggests that James has no markers for dependent use and is not at risk of a withdrawal syndrome. Your management of James may include providing brief intervention using the FRAMES approach in Table 3, harm-minimisation education using the STAYING SAFE proforma in Table 4 and referral to drug and alcohol counselling. Anne is also likely to benefit from being involved in James' care planning and may be provided information about support services available to her.

\footnotetext{
Authors

Vicky Phan BMed, MPH, MPsychiatry, GCertAlc\&DrugSt, Addiction Psychiatry Registrar, Turning Point, Eastern Health, Vic Shalini Arunogiri MBBS (Hons), MSc, MPsychiatry, PhD, FRANZCP, Addiction Psychiatrist and Deputy Clinical Director, Turning Point, Eastern Health, Vic; Senior Lecturer, Monash Addiction Research Centre and Eastern Health Clinical School, Monash University, Vic

Dan I Lubman BSc (Hons), MBChB, PhD, FRANZCP, FAChAM, Director, Turning Point, Eastern Health, Vic; Professor, Monash Addiction Research Centre and Eastern Health Clinical School, Monash University, Vic.dan.lubman@monash.edu

Competing interests: DIL is on an advisory board for Indivior.

Funding: DIL reports grants from National Health and Medical Research Council, Australian Research Council, VicHealth, Victorian Responsible Gambling Foundation, Google and Camurus, outside the submitted work. He also received speaking honoraria from Camurus, Indivior, Lundbeck, AstraZeneca, Janssen-Cilag, Servier and Shire, outside of the submitted work.

Provenance and peer review: Commissioned, externally peer reviewed.
}

\section{References}

1. Manning $V$, Arunogiri S, Frei M, et al. Alcohol and other drug withdrawal: Practice guidelines. 3rd edn. Richmond, Vic: Turning Point, 2018.

2. Degenhardt L, Darke S, Dillon P. GHB use among Australians: Characteristics, use patterns and associated harm. Drug Alcohol Depend 2002;67(1):89-94. doi: 10.1016/s03768716(02)00017-0

3. Dietze PM, Cvetkovski S, Barratt MJ, Clemens S. Patterns and incidence of gammahydroxybutyrate (GHB)-related ambulance attendances in Melbourne, Victoria. Med J Aust 2008;188(12):709-11. doi: 10.5694/j.13265377.2008.tb01851.x.

4. Hillebrand J, Olszewski D, Sedefov R. GHB and its precusor GBL: An emerging trend case study. Lisbon: EMCDDA, 2008. 
5. Australian Institute of Health and Welfare. National drug strategy household survey 2016: Detailed findings. Cat. no. PHE 214. Canberra: AlHW, 2017.

6. Abdulrahim D, Bowden-Jones O. Guidance on the clinical management of acute and chronic harms of club drugs and novel psychoactive substances. London: Novel Psychoactive Treatment UK Network (NEPTUNE), 2015.

7. Lea T, Prestage G, Mao L, Zablotska I, Wit J, Holt M. Trends in drug use among gay and bisexual men in Sydney, Melbourne and Queensland, Australia. Drug Alcohol Rev 2013;32(1):39-46. doi: 10.1111/j.14653362.2012.00494.x

8. Ma R, Perera S. Safer 'chemsex': GPs' role in harm reduction for emerging forms of recreational drug use. Br J Den Pract 2016;66(642):4-5. doi: 10.3399/bjgp16X683029.

9. Bell J, Collins R. Gamma butyrolactone (GBL) dependence and withdrawal. Addiction 2011;106(2):442-47. doi: 10,1111/j.13600443.2010.03145.x.

10. Schep L, Knudsen K, Slaughter RJ Vale JA, Mégarbane B. The clinical toxicology of $\boldsymbol{\gamma}$-hydroxybutyrate, $\mathrm{Y}$-butyrolactone and 1,4-butanediol. Clin Toxicol (Phila) 2012;50(6):458-70. doi: 10.3109/15563650.2012.702218.

11. Gonzalez A, Nutt DJ. Gamma hydroxy butyrate abuse and dependency. J Psychopharmacol 2005;19(2):195-204. doi: 10.1177/0269881105049041.

12. Miotto K, Darakjian J, Basch J, Murray S, Zogg J, Rawson R. Gamma-hydroxybutyric acid: Patterns of use, effects and withdrawal. American J Addict 2001;10(3):232-41. doi: 10.1080/105504901750532111.

13. Galicia M, Nogue S, Miró O. Liquid ecstasy intoxication: Clinical features of 505 consecutive emergency department patients. Emerg Med J 2011;28(6):462-66. doi: 10.1136/emj.2008.068403.

14. Luby S, Jones J, Zalewski A. GHB use in South Carolina. Am J Public Health 1992;82(1):128. doi: 10.2105/ajph.82.1.128-a.

15. Henderson DL, Ginsberg J. Withdrawal, recovery, and long-term sequelae of gamma-butyrolactone dependence: A case report. Am J Addict 2008;17(5):456-57. doi: 10.1080/10550490802266193.

16. Galloway GP, Frederick SL, Staggers FE Jr, Gonzales M, Stalcup SA, Smith DE. Gammahydroxybutyrate: An emerging drug of abuse that causes physical dependence. Addiction 1997;92(1):89-96. doi: 10.1111/j.1360-0443.1997. tb03640.x.

17. Chin R, Sporer KA, Cullison B, Dyer JE, Wu TD Clinical course of gamma-hydroxybutyrate overdose. Ann Emerg Med 1998;31(6):716-22.

18. Miró O, Nogué S, Espinosa G, To-Figueras J, Sánchez M. Trends in illicit drug emergencies: The emerging role of gamma-hydroxybutyrate. J Toxicol Clin Toxicol 2002;40(2):129-35. doi: 10.1081/clt-120004400.

19. Louagie HK, Verstraete AG, De Soete CJ, Baetens DG, Calle PA. A sudden awakening from a near coma after combined intake of gamma-hydroxybutyric acid (GHB) and ethanol. J Toxicol Clin Toxicol 1997;35(6):591-94. doi: 10.3109/15563659709001237.

20. Ingels M, Rangan C, Bellezzo J, Clark R. Coma and respiratory depression following the ingestion of GHB and its precursors: Three cases. J Emerg Med 2000;19(1):47-50. doi: 10.1016/s07364679(00)00188-8.
21. McDonough M, Kennedy N, Glasper A, Bearn J. Clinical features and management of gammahydroxybutyrate (GHB) withdrawal: A review. Drug Alcohol Depend 2004;75(1):3-9. doi: 10.1016/j. drugalcdep.2004.01.012.

22. American Psychiatric Association. Diagnostic and Statistical manual of mental disorders. 5th edn. Arlington, VA: American Psychiatric Association, 2013.

23. Bien TH, Miller WR, Tonigan JS. Brief interventions for alcohol problems: A review. Addiction 1993;88(3):315-33. doi: 10.1111/j.1360-0443.1993. tb00820.x.

24. Global Drug Survey. The High-Way Code: The guide to safer more enjoyable drug use. London: Global Drug Survey. Available at www.globaldrugsurvey.com/wp-content/ uploads/2014/04/The-High-Way-Code_NewDrugs.pdf [Accessed 4 October 2019].

25. Lingford-Hughes AR, Welch S, Peters L, Nutt DJ. BAP updated guidelines: Evidence-based guidelines for the pharmacological management of substance abuse, harmful use, addiction and comorbidity: Recommendations from BAP. J Psychopharmacology 2012;26(7):899-952. doi: 10.1177/0269881112444324. 